\title{
The subspace Nevanlinna interpolation problem and the most powerful unfalsified model
}

\author{
Paolo Rapisarda ${ }^{\mathrm{a}, * .1}$, Jan C. Willems ${ }^{\mathrm{b}}$ \\ a Department of Electrical, Electronic, and Computer Engineering, University of Trieste, Via Valerio, 10, I-34100 Trieste, Italy \\ ${ }^{\mathrm{b}}$ Insitute for Mathematics and Computing Science, University of Groningen, P.O. Box 800 . \\ $9700 \mathrm{AV}$ Groningen, Netherlands
}

Received 5 December 1996; received in revised form 17 March 1997; accepted 11 June 1997

\begin{abstract}
A generalization of the tangential Nevanlinna interpolation problem will be studied from a behavioral point of view. Necessary and sufficient conditions for its solvability and a characterization of all its solutions are derived. These results are obtained by associating to the interpolation data a behavior that enjoys a special structure. (C) 1997 Elsevier Science B.V.
\end{abstract}

Keywords: Interpolation; Linear systems; Polynomial matrices; Quadratic differential forms; Most powerful unfalsified model

\section{Introduction}

Let $N$ distinct points $\lambda_{i}$ in the open right-half complex plane be given together with $N$ subspaces $\mathscr{Y}_{i} \subset \mathbb{C}^{q}$. Assume that there exist nonzero integers $m$ and $p$ with $p+m=q$, such that $k_{i}:=\operatorname{dim}\left(\mathscr{F}_{i}\right) \leqslant m$, $1 \leqslant i \leqslant N$, and

$$
\begin{gathered}
\left\{v=\left(\begin{array}{l}
v_{1} \\
v_{2}
\end{array}\right) \in \mathscr{V}_{i}, \text { with } v_{1} \in \mathbb{C}^{m}, v_{2} \in \mathbb{C}^{p}\right. \\
\text { and } v \neq 0\} \Rightarrow\left\{\mid v_{1}\left\|_{2}>\right\| v_{2} \|_{2}\right\} .
\end{gathered}
$$

We call such a subspace $\mathscr{V}_{i}$ a contractive subspace. Consider now the following problem: given the $N$ pairs $\left(\lambda_{i}, \mathscr{W}_{i}\right)$, find a solynomial $p \times m$ matrix $U$ and a nonsingular $p \times p$ polynomial matrix $Y$ such that

(a) $U$ and $Y$ are left coprime;

(b) $\left(U\left(\lambda_{i}\right)-Y\left(\lambda_{i}\right)\right) v=0 \quad \forall v \in \mathscr{W}_{i}, 1 \leqslant i \leqslant N$;

(c) $\left\|U^{-1} Y\right\|_{H_{\infty}}<1$.

\footnotetext{
* Corresponding author. E-mail: rapisard@univ.trieste.it

' Supported by CNR-NWO and DISC.
}

We will call this problem the subspace Nevanlinna interpolation problem (SNIP in the following), and a pair $(U, Y)$ satisfying (a)-(c) above will be called a solution to the SNIP.

The SNIP is a generalization of the tangential Nevanlinna interpolation problem with simple multiplicities, stated as follows: given $N$ distinct points $\lambda_{i}$ in the open right-half plane and $N$ complex vectors $\left(u_{i} y_{i}\right)^{\mathrm{T}}$ with $u_{i} \in \mathbb{C}^{m}, y_{i} \in \mathbb{C}^{p}, 1 \leqslant i \leqslant N$, find a $p \times m$ rational matrix $G$ such that $G\left(\lambda_{i}\right) u_{i}=y_{i}$, $1 \leqslant i \leqslant N$, and $\|G\|_{H_{\infty}}<1$. The SNIP and the tangential Nevanlinna interpolation problem are connected as follows. Let

$$
\left(\lambda_{i},\left(\begin{array}{c}
u_{i} \\
y_{i}
\end{array}\right)\right)
$$

be the data of the tangential Nevanlinna interpolation problem, and define $\mathscr{V}_{i}$ as the one-dimensional subspace spanned by $\left(u_{i}^{\mathrm{T}} y_{i}^{\mathrm{T}}\right)$. It is easy to see that any solution $(Q, P)$ to the SNIP with data $\left(\lambda_{i}, \mathscr{V}_{i}\right)$ defines a solution $P^{-1} Q$ to the corresponding tangential Nevanlinna interpolation problem. On the other hand, let $G$ be a solution to the tangential Nevanlinna interpola- 
tion problem, and let $P^{-1} Q$ be a left coprime factorization of $G$ with $P$ Hurwitz. It is easy to see that $(Q, P)$ is a solution of the SNIP with data $\left(\lambda_{i}, \mathscr{Y}_{i}\right)$.

The literature on Nevanlinna-type interpolation problems is vast. For the scalar case ( $m=p=1$ and $k_{i}=1$ for $i \in \underline{N}$ ), necessary and sufficient conditions for solvability and an iterative algorithm for the computation of a solution have been stated in $[9,10]$; the tangential version of the problem was first solved in [4]. Many different methods of solution have been devised; we refer the reader to the monograph [2], which gives a thorough exposition of interpolation theory for rational matrix functions and a copious list of references. One reason why Nevanlinna-type interpolation problems have been investigated is that they are connected with system-, circuit- and control-theoretic issues as interpolation with positive-real functions [16], model approximation [5], electrical power transfer [7], robust stabilization [8], and model matching in the $H_{\infty}$-norm [3].

In this paper we deal with the SNIP from a behavioral point of view. We give necessary and sufficient conditions for its solvability and we characterize all its solutions. Two notions developed in the behavioral context will be instrumental in this: the concept of most powerful unfalsified model and the calculus of quadratic differential forms. In order to make the paper as self-contained as possible, in Sections 2 and 3 we illustrate these notions, restricting our exposition to the bare essentials; further details can be found in $[11,14]$. In Section 4 necessary and sufficient conditions for the existence of a solution to the SNIP are stated, while in Section 5 a characterization of all solutions to the SNIP is given.

We use the following notation. Given a set $X, 2^{X}$ denotes the power set of $X, \mathbb{N}, \mathbb{R}$ and $\mathbb{C}$ denote the sets of natural, of real and of complex numbers, respectively. $\mathbb{C}_{+}$denotes the open right-half of the complex plane and $\mathbb{C}_{+}^{0}$ the closed right-half plane. Given $n \in \mathbb{N}, \underline{n}$ denotes the set $\{i \in \mathbb{N} \mid 1 \leqslant i \leqslant n\} .\left(\mathbb{C}^{q}\right)^{\mathbb{R}}$ is the set of all maps from $\mathbb{R}$ to $\mathbb{C}^{q} . \mathscr{C}^{\infty}\left(\mathbb{R}, \mathbb{C}^{q}\right)$ denotes the set of infinitely differentiable $q$-valued complex functions, and $\mathscr{L}_{2}\left(\mathbb{R}, \mathbb{C}^{q}\right)$ the set of square-integrable $q$-valued complex functions. $\|\cdot\|_{2}$ denotes the 2-norm; $\|\cdot\|_{\infty},\|\cdot\|_{H_{\infty}}$, and $\|\cdot\|_{\mathscr{Z}_{2}}$ respectively the $\infty-, H_{\infty}$, and $\mathscr{L}_{2}$-norms. We denote with $\sigma^{\tau}:\left(\mathbb{C}^{q}\right)^{\mathbb{R}} \rightarrow\left(\mathbb{C}^{q}\right)^{\mathbb{R}}$ the $\tau$-shift defined by $\left(\sigma^{\tau} f\right)(t):=f(t+\tau) \forall t \in \mathbb{R}$. $\exp _{;}: \mathbb{R} \rightarrow \mathbb{C}$ is defined as $\exp _{\gamma}(t):=\mathrm{e}^{\hat{\lambda} t}$. A linear, shift-invariant, differential behavior with infinitely differentiable $q$-dimensional complex trajectories is a subset $\mathscr{B} \subseteq \mathscr{C}^{\infty}\left(\mathbb{R}, \mathbb{C}^{q}\right)$ enjoying the following property: there exists a polynomial matrix $R$ with $q$ columns such that $\not B=\left\{w \in \mathscr{C}^{\infty}\left(\mathbb{R}, \mathbb{C}^{q}\right) \mid R(\mathrm{~d} / \mathrm{d} t) w=0\right\}$. The set of all such linear, shift-invariant, differential behaviors with infinitely differentiable $q$-dimensional complex trajectories will be denoted as $\mathscr{L}\left(\mathbb{R}, \mathbb{C}^{4}\right)$. The set of polynomials with complex coefficients in the indeterminate $\xi$ is denoted by $\mathbb{C}[\xi]$, and the set of polynomial $q_{1} \times q_{2}$ matrices with complex coefficients in the indeterminate $\xi$ is denoted by $\mathbb{C}^{q_{1} \times q_{2}}[\xi]$. The map $*: \mathbb{C}^{q_{1} \times q_{2}}[\xi] \rightarrow \mathbb{C}^{q_{2} \times q_{1}}[\xi]$ is defined as follows. Let $R(\xi)=\sum_{i=0}^{l} R_{i} \xi^{i}$; then $R^{*}(\xi):=\sum_{i=0}^{l} \bar{R}_{i}^{\mathrm{T}}(-\zeta)^{i}$, or, more compactly, $R^{*}(\xi)=\bar{R}^{\mathrm{T}}(-\xi)$, with ${ }^{-}$complex conjugate. Given $r$ matrices $A_{i} \in \mathbb{C}^{k_{i} \times k}, i \in \underline{r}$, $\operatorname{col}\left(A_{i}\right)_{i \in \underline{r}}$ denotes the $\left(\sum_{i=1}^{r} k_{i}\right) \times k$ matrix

$\left(\begin{array}{c}A_{1} \\ \vdots \\ A_{r}\end{array}\right)$.

Given $r$ matrices $A_{i} \in \mathbb{C}^{k_{i} \times k_{i}}, \quad i \in \underline{r}, \operatorname{diag}\left(A_{i}\right)_{i \in \underline{r}}$ denotes the $r \times r$ block-matrix with the $A_{i}$ 's on the main diagonal and zero blocks elsewhere. $J_{k_{1}, k_{2}} \in \mathbb{C}^{\left(k_{1}+k_{2}\right) \times\left(k_{1} \times k_{2}\right)}$ denotes the signature matrix $\left(\begin{array}{cc}I_{k_{1}} & 0_{k_{1}} \times k_{2} \\ 0_{k_{2} \times k_{1}} & -I_{k_{2}}\end{array}\right)$.

\section{The most powerful unfalsified model}

The notion of most powerful unfalsified model has been introduced in [12] in the context of exact modeling of time series. Let $w_{i}: \mathbb{R} \rightarrow \mathbb{C}^{q}, i \in \underline{S}$, be given (continuous-time) time series. For the purposes of this paper, we assume that $w_{i} \in \mathscr{C}^{\infty}\left(\mathbb{R}, \mathbb{C}^{q}\right)$ for every $i \in \underline{S}$. Let $\mathscr{A} \subseteq 2^{\left(\mathbb{C}^{4}\right)^{\mathrm{F}}}$ be a class of models, the choice of which reflects the assumptions that the modeler wishes to make on the structure of the phenomenon that produced the $w_{i}$ 's; for example, if models described by linear constant-coefficient differential equations are sought, then $\mathscr{M}=\mathscr{L}\left(\mathbb{R}, \mathbb{C}^{q}\right) . \mathscr{B} \in \mathscr{M}$ is an unfalsified model for the data set $\left\{w_{i}\right\}_{i \in \underline{S}}$ if $w_{i} \in \mathscr{B} \forall i \in \underline{S}$. For many choices of $\mathscr{M}$ an unfalsified model for $\left\{\bar{w}_{i}\right\}_{i \in \underline{S}}$ always exists: for example, if $. \mathscr{H}=2^{\left(\mathbb{C}^{4}\right)^{\mathbb{R}}}$, one can take as model for the $w_{i}$ 's the behavior consisting of all complex $q$-dimensional trajectories. Such a model is, however, of little practical use: it is unfalsified not only by the data, but by any set of trajectories. In this sense, a good model is a behavior which is unfalsified by the data and which explains as little else as possible. We formalize this intuitive notion as 
follows. We call r $^{*}$ the most powerful unfalsified model (MPUM) in $\mathscr{U}$ for $\left\{w_{i}\right\}_{i \in S}$ if $w_{i} \in \mathscr{B}^{*} \forall i \in \underline{S}$, and $\left\{w_{i} \in \mathscr{B}^{\prime}, \forall i \in \underline{S}\right.$ and $\left.\mathscr{B}^{\prime} \in \mathscr{M}\right\} \Rightarrow\left\{\mathscr{B}^{*} \subseteq \mathscr{B}^{\prime}\right\}$. Note that the MPUM need not exist; however, for many interesting choices of $\mathscr{U}$ the MPUM exists and is unique; an instance of this is $\mathscr{H}=\mathscr{L}\left(\mathbb{R}, \mathbb{C}^{q}\right)$.

Let us now turn to the SNIP and its connections with behavioral modeling. We associate with each pair $\left(\hat{\lambda}_{i}, \hat{V}_{i}\right)$ the subspace

$\mathscr{F}_{i} \exp _{\lambda_{i}}:=\left\{v \exp _{\lambda_{j}} \mid v \in \mathscr{V}_{i}\right\} \subset \mathscr{L}\left(\mathbb{R}, \mathbb{C}^{p+m}\right)$.

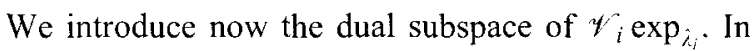
order to do this, let us define $\mathscr{V}_{i}^{\perp}$ first as

$\mathscr{V}_{i}^{\perp}:=\left\{w \in \mathbb{C}^{m+p} \mid \bar{w}^{\mathrm{T}} J v=0 \quad \forall v \in \mathscr{V}_{i}\right\}$,

where here and in the rest of the paper $J:=J_{m, p}$. Note that if $\mathscr{V}_{i}$ is contractive, $\mathscr{V}_{i}{ }^{\perp}$ is uniquely defined, as shown in the next proposition.

Proposition 2.1. Let $\mathscr{F}$ be a contractive subspace of $\mathbb{C}^{m+p}$. Then $\mathscr{V} \perp \oplus \mathscr{Y}=\mathbb{C}^{p+m}$.

Proof. Observe first that $\mathscr{Y} \cap \mathscr{V}^{\perp}=\{0\}$. In fact, let $w \in \mathscr{V} \cap \mathscr{V}^{\perp}$, with $w$ partitioned as

$\left(\begin{array}{l}w_{1} \\ w_{2}\end{array}\right)$

where $w_{1} \in \mathbb{C}^{m}, w_{2} \in \mathbb{C}^{p}$. Since $\bar{w}^{-T} J w=0$, there follows $\left\|w_{1}\right\|_{2}-\left\|w_{2}\right\|_{2}=0$; but since the only vector in $\mathscr{V}$ satisfying $\left\|w_{1}\right\|_{2}-\left\|w_{2}\right\|_{2}=0$ is the zero vector, there follows $w=0$.

Assume now that $\left\{v_{i}\right\}_{i \in \underline{k}}$ is a basis for $\mathscr{V}$, and note that the full column rank matrix $V:=\left(v_{1} \ldots v_{k}\right)$ is such that $\operatorname{Im}(V)=\mathscr{V}$. Observe also that $w$ belongs to $\mathscr{F}^{\perp}$ if and only if $\ddot{w}^{\mathrm{T}} J V=0$, i.e. if and only if $w$ is orthogonal (in the Elclidean inner product sense) to all columns of $J V$. Evidently, this requirement specifies a unique $(m+p-k)$-dimensional subspace $\mathscr{V}^{\perp} \subset \mathbb{C}^{p+m}$ such that $\mathscr{V} \oplus \mathscr{V}^{L}=\mathbb{C}^{p+m}$. The claim is therefore proved.

We define the dual subspace of $\mathscr{V}_{i} \exp _{i_{i}}$ as

$\mathscr{T}_{i}^{\perp} \exp _{-\bar{i}_{i}}:=\left\{v \exp _{-\bar{\lambda}_{i}} \mid v \in \mathscr{Y}_{i}^{\perp}\right\}$.

Define also the data set $\mathscr{Z}$ as

$\mathscr{D}:=\bigcup_{i \in \underline{N}} \mathscr{\mathscr { V }}_{i} \exp _{i, i} \cup \mathscr{W}_{i}^{\perp} \exp _{-\bar{\lambda}_{i}}$.

As we will see in Sections 4 and 5, the MPUM for $\mathscr{D}$ in the model class $\mathscr{L}\left(\mathbb{R}, \mathbb{C}^{q}\right)$ is instrumental in stating necessary and sufficient conditions for the solvability of the NIP and for characterizing all its solutions. We now describe the main features of this MPUM and we illustrate an algorithm to compute a kernel representation for it.

A polynomial exponential time series is a trajectory of the form

$w_{i}(t)=\sum_{j=0}^{v_{i}} v_{i j} \frac{t^{j}}{j !} \exp _{i_{i}}(t), \quad v_{i j} \in \mathbb{C}^{q}, \quad i \in \underline{N}$.

Note that $\mathscr{P}$ defined in Eq. (5) consists of linear combinations of polynomial exponential trajectories with $v_{i}=0, i \in \underline{N}$.

The problem of computing the MPUM in $\mathscr{L}\left(\mathbb{R}, \mathbb{C}^{q}\right)$ for a set of polynomial exponential trajectories has been considered in [1]; in the following we summarize those results which are more relevant to the SNIP. It can be proved [1, p. 1787] that in the case of polynomial exponential time series the MPUM is an autonomous (i.e. finite-dimensional) behavior, and therefore can be represented in kernel form by a nonsingular polynomial matrix $R$; the dimension of the MPUM equals the degree of the determinant of $R$. It can be proved (see $[1$, p. 1789]) that this determinant has all its roots at the characteristic frequencies $\lambda_{i}$. To illustrate how the multiplicity of $\lambda_{i}$ as a root of $\operatorname{det}(R)$ depends on the trajectories $w_{i}$ to be modeled, we consider the case of interest to us in which the $\hat{\lambda}_{i}$ 's are distinct and the MPUM for the set $\left\{w_{i j}=v_{i j} \exp _{\lambda_{i}} \mid j \in \underline{k_{i}}\right.$ and $i \in \underline{N}\}$ is sought. Let $V_{i}:=\left(\begin{array}{lll}v_{i 1} & \ldots & v_{i k_{i}}\end{array}\right)$; it can be shown (see [1, p. 1788]) that the dimension of the MPUM in this case equals $\sum_{i=1}^{N} \operatorname{Rank}\left(V_{i}\right)$.

Note that a kernel representation of the MPUM is not unique: in fact, all other representations with $R$ square can be obtained by premultiplication of $R$ by a unimodular matrix (see [13, p. 263]). Note also that all unfalsified models $\mathscr{B}^{\prime} \in \mathscr{L}\left(\mathbb{R}, \mathbb{C}^{q}\right)$ can be represented in kernel form by premultiplication of $R$ by a suitable polynomial matrix (see [11, p. 565]).

In [13] a recursive procedure for computing a MPUM in $\mathscr{L}\left(\mathbb{R}, \mathbb{C}^{q}\right)$ for a finite set of polynomialexponential data has been given. We now illustrate it for the data set $\left\{v_{i} \exp _{i_{i}}\right\}_{i \in \underline{S} \text {. }}$.

Define the 0th step model as $M_{0}(\xi):=I_{q}$, and the $i$ th step model as $M_{i}(\xi):=E_{i}(\xi) M_{i-1}(\xi), i \in \underline{S}$, where $E_{i}(\xi)$ represents a MPUM for the $i$ th error trajectory $\varepsilon_{i}:=M(\mathrm{~d} / \mathrm{d} t) v_{i} \exp _{j_{i}}$. Note that the MPUM for a data set consisting of only one trajectory $v \exp _{j, v} \neq 0$, can be computed as $\left(v v^{\mathrm{T}} /\|v\|_{2}^{2}\right) \mathrm{d} / \mathrm{d} t-\dot{\lambda} I_{q}$. It can be shown (see [13, p. 289]) that $M_{S}$ represents the MPUM for $\left\{v_{i} \exp _{j_{i}}\right\}_{i \in \underline{S}}$. In Section 4 we will use a modification 
of this procedure to prove the existence of a special representation of the MPUM for $\mathscr{Z}$.

\section{Quadratic differential forms}

A $q \times q$ symmetric two-variable polynomial matrix in the indeterminates $\zeta$ and $\eta$ is an expression

$\Phi(\zeta, \eta)=\sum_{h, k=0}^{L} \Phi_{h, k} \zeta^{h} \eta^{k}$

where the complex matrices $\Phi_{h, k}$ satisfy $\Phi_{h, k}=\bar{\Phi}_{k, h}^{\mathrm{T}}$. We associate to Eq. (7) the quadratic differential form (QDF) $Q_{\Phi}: \mathscr{C}^{\infty}\left(\mathbb{R}, \mathbb{C}^{q}\right) \rightarrow \mathscr{C}^{\infty}(\mathbb{R}, \mathbb{R})$ defined as

$Q_{\Phi}(w):=\sum_{h, k=0}^{L}\left(\frac{\mathrm{d}^{h} \bar{w}}{\mathrm{~d} t^{h}}\right)^{\mathrm{T}} \Phi_{h, k} \frac{\mathrm{d}^{k} w}{\mathrm{~d} t^{k}}$.

A very convenient aspect of the association of QDFs with two-variable polynomial matrices is that a calculus of QDFs is easily developed and has far-reaching applications (see [14, 15]).

In many applications it is necessary to compute the integral of a QDF. To ensure that such an integral exist, we assume that the argument of the QDF is an infinitely differentiable function of compact support. We will denote the set of infinitely differentiable functions from $\mathbb{R}$ to $\mathbb{C}^{q}$ with compact support as $\mathscr{T}\left(\mathbb{R}, \mathbb{C}^{q}\right)$. In the context of integrals of QDFs, an important role in this paper is played by the notion of average- and half-line nonnegativity of a QDF. $Q_{\Phi}$ is said to be average-nonnegative (denoted as $\int Q_{\phi} \geqslant 0$ ) if for all trajectories $w \in \mathscr{D}\left(\mathbb{R}, \mathbb{C}^{q}\right)$ there holds

$\int_{-\infty}^{+\infty} Q_{\phi}(w) \mathrm{d} t \geqslant 0$

Define the map $\partial: \mathbb{C}^{q \times q}[\zeta, \eta] \rightarrow \mathbb{C}^{q \times q}[\xi]$ as $\hat{\partial} \Phi(\xi)$ $:=\Phi(-\xi, \xi)$. It can be proved (see $[14$, p. 21]) that $\int Q_{\Phi} \geqslant 0$ if and only if the Hermitian matrix $\partial \Phi(\mathrm{i} \omega)$ is nonnegative definite for all $\omega \in \mathbb{R}$. A QDF $Q_{\Phi}$ is called average positive, denoted by $\int Q_{\phi}>0$, if Eq. (9) holds and $\left\{\int_{-\infty}^{\infty} Q_{\phi}(w) \mathrm{d} t=0\right\} \Rightarrow\{w=0\}$. It can be proved (see [14, p. 21]) that $\int Q_{\Phi}>0$ if and only if $\partial \Phi(\mathrm{i} \omega) \geqslant 0 \forall \omega \in \mathbb{R}$ and $\operatorname{det}(\delta \Phi) \neq 0$.

A related notion is that of half-line nonnegativity (positivity); $Q_{\Phi}$ is called half-line nonnegative, denoted as $\int^{0} Q_{\Phi} \geqslant 0$, if

$$
\int_{-\infty}^{0} Q_{\Phi}(w) \mathrm{d} t \geqslant 0 \quad \forall w \in \mathscr{D}\left(\mathbb{R}, \mathbb{C}^{q}\right)
$$

and half-line positive, denoted as $\int^{0} Q_{\Phi}>0$, if Eq. (10) holds and $\left\{\int_{-\infty}^{0} Q_{\phi}(w) \mathrm{d} t=0\right\} \Rightarrow\{w=0\}$.

In [14] the Pick matrix associated with a QDF has been introduced as a tool for testing half-line positivity; in Section 4 of the present paper we will use a generalization of this concept to give necessary and sufficient conditions for the solvability of the SNIP. In order to define the Pick matrix associated with a QDF, we have to introduce the notion of semi-simplicity of a polynomial matrix. Let $F \in \mathbb{C}^{q \times q}[\xi]$ be nonsingular; then it is semi-simple if $\forall \lambda \in \mathbb{C}$ the dimension of $\operatorname{Ker}(F(\lambda))$ is equal to the multiplicity of $\lambda$ as a root of $\operatorname{det}(F)$. For the purposes of the present paper, we introduce the notion of Pick matrix associated to a QDF in the semi-simple case only. Let $\phi \in \mathbb{C}^{q \times q}[\zeta, \eta]$ be a symmetric two-variable polynomial matrix. Assume that $\operatorname{det}(\delta \Phi)$ has no roots on the imaginary axis, and let $\lambda_{i} \in \mathbb{C}_{+}, i \in \underline{r}$, be its right-half plane roots. Let $a_{i} \in \mathbb{C}^{q}, i \in \underline{r}$, be linearly independent vectors such that $\partial \Phi\left(\lambda_{i}\right) a_{i}=0$ and such that the $a_{k}$ 's associated with the same $\lambda_{i}$ span $\operatorname{Ker}\left(\partial \Phi\left(\lambda_{i}\right)\right)$. The Pick matrix of $\Phi$ is the Hermitian $r \times r$ matrix defined by

$T_{\phi}:=\left(\frac{\bar{a}_{i}^{\mathrm{T}} \Phi\left(\bar{\lambda}_{i}, \hat{i}_{j}\right) a_{j}}{\bar{\lambda}_{i}+i_{j}}\right)_{i, j \in \underline{r}}$.

Finally, let us introduce the notion of dual of a QDF (see [14, p. 34]). Let $D \in \mathbb{C}^{m \times m}[\xi]$ and $N \in \mathbb{C}^{p \times m}[\xi]$ be right coprime matrices, and let $Q \in \mathbb{C}^{p \times m}[\xi]$ and $P \in \mathbb{C}^{p \times p}[\xi]$ be left coprime matrices such that $Q D-P N=0$. Introduce the two-variable polynomials $\Phi(\zeta, \eta)=\bar{D}^{\mathrm{T}}(\zeta) D(\eta)-\bar{N}^{\mathrm{T}}(\zeta) N(\eta)$ and $\Psi(\zeta, \eta)=Q(-\zeta) \bar{Q}^{\mathrm{T}}(-\eta)-P(-\zeta) \bar{P}^{\mathrm{T}}(-\eta)$. The QDF $Q_{\Psi}$ will be called the dual of the QDF $Q_{\Phi}$. It is possible to show (see [14, p. 36]) that the positivity properties of $Q_{\Psi}$ and $Q_{\Phi}$ are related: in fact, $Q_{\Phi}$ is average nonnegative (positive) if and only if $Q_{\Psi}$ is average nonpositive (negative).

\section{Necessary and sufficient conditions for the solvability of the SNIP}

In this section we state two necessary and sufficient conditions for the existence of a solution to the SNIP. The first condition is analogous to the classical one, namely the positive definiteness of the Pick matrix associated with the data (see [2, p. 387], for the case $k_{i}=1$ for $i \in \underline{N}$ ). The second one is new, and relates the SNIP to special representations of the MPUM of the data set $\mathscr{D}$ defined in Eq. (5). 
Before stating the main result of this section, let us introduce the Pick matrix of the data $\left(\lambda_{i}, \mathscr{V}_{i}\right)_{i \in N}$. Assume that a full column rank matrix $V_{i} \in \mathbb{C}^{q \times \bar{k}_{i}}$ is given such that $\operatorname{Im}\left(V_{i}\right)=\mathscr{V}_{i}$, for every $i \in \underline{N}$. We will call the Hermitian $\left(\sum_{i=1}^{N} k_{i}\right) \times\left(\sum_{i=1}^{N} k_{i}\right)$ matrix

$$
T_{\{\gamma,\}_{i \in \underline{X}}}:=\left(\frac{\bar{V}_{i}^{\mathrm{T}} J V_{i}}{\bar{\lambda}_{i}+\lambda_{j}}\right)_{i, j \in \underline{N}}
$$

the Pick matrix of the data. Note that $T_{\{\}_{\}} \in \in}$ depends on the particular basis matrices $V_{i}$ chosen, but that its positive definiteness depends only on the subspaces $\mathscr{V}_{i}$.

We are now ready to state the main result of this section.

Theorem 4.1. The following three statements are equivalent:

1. The Hermitian matrix $T_{\left\{1_{i}\right\}_{\in \underline{X}}}$ is positive definite;

2. The MPUM for $\mathscr{D}$ has a kernel representation induced by a matrix of the form

$$
R:=\left(\begin{array}{cc}
-D^{*} & N^{*} \\
Q & -P
\end{array}\right)
$$

where $D \in \mathbb{C}^{m \times n}[\xi], N \in \mathbb{C}^{p \times m}[\xi], Q \in \mathbb{C}^{p \times m}[\xi]$, $P \in \mathbb{C}^{p \times p}[\xi]$ saitisfy the following properties:
(a) $D$ and $P$ are nonsingular;
(b) $Q D-P N=0$;
(c) $P$ is Hurwitz;
(d) $R J R^{*}=p p^{*} J=R^{*} J R$ with $p \in \mathbb{C}[\xi]$ a Hurwitz polvnomial;
(e) $\left\|P^{-1} Q\right\|_{H_{\infty}}<1$;
(f) $\left\|N^{*} P^{-1}\right\|_{H_{\infty}}<1$;

3. There exists a solution to the SNIP.

Proof. We will run the circle $(1) \Rightarrow(2) \Rightarrow(3) \Rightarrow$ (1).

Let us first prove $(1) \Rightarrow(2)$. We will prove this by induction on the number $N$ of subspaces $\mathscr{Y}_{i}$ to interpolate.

For $N=1$, partition the basis matrix $V_{1}$ for $\mathscr{V}_{1}$ as

$$
V_{1}=\left(\begin{array}{l}
V_{11} \\
V_{12}
\end{array}\right)
$$

with $V_{11} \in \mathbb{C}^{m \times k_{1}}$ and $V_{12} \in \mathbb{C}^{p \times k_{1}}$, and consider the model $\mathscr{B}_{1}$ represented in kernel form by

$$
R_{1}(\xi):=\left(\xi+\bar{\lambda}_{1}\right) I_{p+m}-V_{1} T_{\left\{y_{1}\right\}}^{-1} \bar{V}_{1}^{\mathrm{T}} J .
$$

For future reference, partition Eq. (14) according to the partition of $V_{1}$ as

$$
\begin{aligned}
& \left(\begin{array}{cc}
-D_{1}^{*} & N_{1}^{*} \\
Q_{1} & -P_{1}
\end{array}\right):= \\
& \left(\begin{array}{cc}
\left(\xi+\bar{\lambda}_{1}\right) I_{m}-V_{11} T_{\{, 1\}}^{-1} \bar{V}_{11}^{\mathrm{T}} & V_{11} T_{\{1,\}}^{-1} \bar{V}_{12}^{\mathrm{T}} \\
-V_{12} T_{\{, 1\}}^{-1} \bar{V}_{11}^{\mathrm{T}} & \left(\xi+\bar{\lambda}_{1}\right) I_{p}+V_{12} T_{\left\{y_{1}\right\}}^{-1} \bar{V}_{12}^{\mathrm{T}}
\end{array}\right) .
\end{aligned}
$$

Note that

$$
\begin{aligned}
\left(\frac{\mathrm{d}}{\mathrm{d} t}\right. & \left.+\bar{\lambda}_{1}\right) V_{1} \exp _{\lambda_{1}}-V_{1} T_{\left\{\psi_{i}\right\}}^{-1} \bar{V}_{1}^{\mathrm{T}} J V_{1} \exp _{\lambda_{1}} \\
= & \left(\bar{\lambda}_{1}+\bar{\lambda}_{1}\right) V_{1} \exp _{\lambda_{1}} \\
& -V_{1}\left(\frac{\bar{V}_{1}^{\mathrm{T}} J V_{1}}{\lambda_{1}+\bar{\lambda}_{1}}\right)^{-1}\left(\bar{V}_{1}^{\mathrm{T}} J V_{1}\right) \exp _{\lambda_{1}}=0
\end{aligned}
$$

Note also that if $Z_{1}$ is a $q \times\left(m+p-k_{1}\right)$ matrix such that $\operatorname{Im}\left(Z_{1}\right)=\mathscr{\mathscr { V }} \perp$, there holds

$$
\begin{aligned}
& \left(\frac{\mathrm{d}}{\mathrm{d} t}+{\overline{i_{1}}}_{1}\right) Z_{1} \exp _{-\bar{i}_{1}} \\
& \quad-V_{1} T_{\left\{t_{1}\right\}}^{-1} \bar{V}_{1}^{\mathrm{T}} J Z_{1} \exp _{-\bar{\lambda}_{1}}=0 .
\end{aligned}
$$

Therefore, $\mathscr{B}_{1} \supseteq \mathscr{F}_{1} \exp _{i_{1}}$ and $\mathscr{B}_{1} \supseteq \mathscr{V}_{1}^{\perp} \exp {\overline{\lambda_{1}}}_{1}$. In order to prove that $\mathscr{B}_{1}$ is the MPUM, observe that the determinant of Eq. (14) has degree $p+m$, and therefore $\mathscr{B}_{1}$ contains $p+m$ independent trajectories. Since $\operatorname{dim}\left(\mathscr{V}_{1} \exp _{\hat{\lambda}_{1}} \oplus \mathscr{V}_{1}^{\perp} \exp _{-\bar{j}_{1}}\right)=m+p$, the claim is proved.

We now prove that the representation given by Eq. (15) satisfies (2a)-(2f). Since $D_{1}$ and $P_{1}$ in Eq. (15) are row proper, they are nonsingular. $Q_{1} D_{1}-P_{1} N_{1}=0$ follows from straightforward manipulations. To prove that $P_{1}$ is Hurwitz, assume by contradiction that there exists a $\mu \in \mathbb{C}_{+}^{0}$ and a nonzero vector $v \in \mathbb{C}^{p}$ such that

$P_{1}(\mu) v=\left(\mu+\bar{\lambda}_{1}\right) v+V_{12} T_{\{y, 1}^{-1} \bar{V}_{12}^{\mathrm{T}} v=0$.

Multiplying Eq. (18) on the left by $\bar{c}^{\mathrm{T}}$, we obtain

$\left(\mu+\bar{\lambda}_{1}\right)\|v\|_{2}^{2}+\bar{v}^{\mathrm{T}} V_{12} T_{\left\{y_{i}\right\}}^{-1} \bar{V}_{12}^{\mathrm{T}} v=0$.

From the assumption that $T_{\left\{y_{1}\right\}}$ is positive definite follows that the second term in Eq. (19) is strictly greater than zero; it is easy to see that this implies $\operatorname{Re}(\mu)<0$, a contradiction with $\mu \in \mathbb{C}_{+}^{0}$. 
Let us now prove ( $2 \mathrm{~d}$ ). Observe that

$$
\begin{aligned}
R_{1} J R_{1}^{*}= & {\left[\left(\xi+\bar{\lambda}_{1}\right) I_{p+m}-V_{1} T_{\{y, 1\}}^{-1} \bar{V}_{1}^{\mathrm{T}} J\right] } \\
& \times J\left[\left(-\xi+\lambda_{1}\right) I_{p+m}-J V_{1} T_{\{y ;\}}^{-1} \bar{V}_{1}^{\mathrm{T}} J\right] \\
= & \left(\xi+\bar{\lambda}_{1}\right)\left(-\xi+\dot{\lambda}_{1}\right) J-\left(\bar{\lambda}_{1}+\bar{\lambda}_{1}\right) V_{1} T_{\{y i j\}}^{-1} \bar{V}_{1}^{\mathrm{T}} \\
& +\left(\hat{\lambda}_{1}+\bar{\lambda}_{1}\right) V_{1} T_{\{t i\}}^{-1} \bar{V}_{1}^{\mathrm{T}} \\
= & \left(\xi+\bar{\lambda}_{1}\right)\left(-\xi+\lambda_{1}\right) J .
\end{aligned}
$$

The second equality of ( $2 \mathrm{~d})$ can be proved analogously.

To prove (2e), observe that from (2a) and (2b) it follows that $P_{1}^{-1} Q_{1}=N_{\mathrm{l}} D_{1}^{-1}$. In order to prove $\left\|P_{1}^{-1} Q_{1}\right\|_{\infty}<1$, we will therefore consider the QDF induced by $\Psi(\zeta, \eta)=\bar{D}_{1}^{\mathrm{T}}(\zeta) D_{1}(\eta)-$ $\bar{N}_{1}^{\mathrm{T}}(\zeta) N_{\mathrm{I}}(\eta)$ and prove that $\delta \Psi(\mathrm{i} \omega)=D_{1}^{*}(\mathrm{i} \omega) D_{1}(\mathrm{i} \omega)$ - $N_{1}^{*}(\mathrm{i} \omega) N_{1}(\mathrm{i} \omega)>0$ for every $\omega \in \mathbb{R}$. Note that $D_{1}^{*} D_{1}-N_{1}^{*} N_{1}$ is the $(1,1)$-block of $R_{1} J R_{1}^{*}$ and, by (2d), on the imaginary axis it equals

$\left(-\mathrm{i} \omega+\bar{\lambda}_{1}\right)\left(\mathrm{i} \omega+\bar{\lambda}_{1}\right) I_{m}$,

which is positive definite for every $\omega \in \mathbb{R}$. To prove claim (2f), note that $N_{1}(\mathrm{i} \omega) N_{1}^{*}(\mathrm{i} \omega)-P_{1}^{*}(\mathrm{i} \omega) P_{1}(\mathrm{i} \omega)$ is the $(2,2)$ block of $R_{1}(\mathrm{i} \omega)^{*} J R_{\mathrm{l}}(\mathrm{i} \omega)$ and that by $(2 \mathrm{~d})$ it is negative definite for all $\omega \in \mathbb{R}$. This concludes the proof of $(2 a)-(2 f)$ for the representation given by Eq. (14) of the MPUM for $N=1$.

Let us now assume that the claim $(1) \Rightarrow(2)$ holds for a number $j$ of subspaces to interpolate, $j \in \underline{N-1}$. In order to prove the claim for $N$ subspaces we will proceed as follows. We have shown above that there exists a representation $R_{1}$ of the MPUM for $\mathscr{F}_{1} \exp _{i_{1}} \oplus$ $\mathscr{V}_{1}^{\perp} \exp _{-i_{1}}$ that satisfies (2a)-(2f). We will show that the Pick matrix of the errors $\left\{R_{1}(\mathrm{~d} / \mathrm{d} t) \mathscr{V}_{i} \exp _{j_{i}}\right\}_{2 \leqslant i \leqslant N}$ associated with this representation is positive definite. Then we will apply the inductive assumption and conclude that a representation $R^{\prime}$ of the MPUM for the errors satisfying (2a)-(2f) exists. Combining the representations of the two MPUMs as $R^{\prime} R_{\mathrm{J}}$ we obtain a representation of the MPUM for $\mathscr{D}$, and we will show that it satisfies (2a)-(2f).

Assume now that a representation given by Eq. (15) of the MPUM for $\mathscr{V}_{1} \exp _{i_{1}} \odot \mathscr{V}_{1}^{\perp} \exp _{-i_{1}}$ has been computed, satisfying $(2 a)-(2 f)$. The error subspaces of this model are

$$
V_{i}^{\prime}:=\left(\lambda_{i}+\bar{i}_{1}\right) V_{i}-V_{1} T_{\left\{y_{1}\right\}}^{-1} \bar{V}_{1}^{\mathrm{T}} J V_{i} .
$$

We now prove that the Pick matrix $T_{\left\{y^{\prime}\right\}_{2 \leqslant i \leqslant 1}}$ is positive definite. Note first that for $2 \leqslant i \leqslant N, 2 \leqslant j \leqslant N$ the $(i-1, j-1)$ th block element of $T_{\left\{y_{i}\right\}_{2 \leqslant \infty}}$ is

$$
\begin{aligned}
\frac{\bar{V}_{i}^{\prime \mathrm{T}} J V_{j}^{\prime}}{\overline{\bar{\lambda}}_{i}+\lambda_{j}}= & {\left[\left(\bar{\lambda}_{i}+i_{1}\right) \bar{V}_{i}^{\mathrm{T}}-\bar{V}_{i}^{\mathrm{T}} J V_{1} T_{\left\{t_{i}\right\}}^{-1} \bar{V}_{1}^{\mathrm{T}}\right] } \\
& \times J\left[\left(\bar{\lambda}_{j}+\bar{\lambda}_{1}\right) V_{j}-V_{1} T_{\left\{y_{i}\right\}}^{-1} \bar{V}_{1}^{\mathrm{T}} J V_{j}\right] \\
& \times\left(\bar{\lambda}_{i}+\bar{\lambda}_{j}\right)^{-1} .
\end{aligned}
$$

Easy computations show that Eq. (23) equals

$$
\frac{\left(\bar{\lambda}_{i}+\bar{\lambda}_{1}\right)\left(\lambda_{j}+\bar{\lambda}_{1}\right)}{\bar{\lambda}_{i}+\lambda_{j}} \bar{V}_{i}^{\mathrm{T}} J V_{j}-\bar{V}_{i}^{\mathrm{T}} J V_{1} T_{\left\{f_{1\}}\right.}^{-1} \bar{V}_{1}^{\mathrm{T}} J V_{j} .
$$

Partition now $T_{\left\{x_{i}\right\}_{i \in \underline{I}}}$ as

$$
\left(\begin{array}{cc}
T_{\{y, 1\}} & \bar{b}^{\mathrm{T}} \\
b & T_{\{y,\}_{2} \leqslant i \leqslant N}
\end{array}\right)
$$

with $b=\operatorname{col}\left(\bar{V}_{i}^{\mathrm{T}} J V_{1} / \overline{\bar{\lambda}}_{i}+\lambda_{1}\right)_{2 \leqslant i \leqslant N}$, and define $\Delta:=\operatorname{diag}\left(\left(\bar{\lambda}_{i}+\lambda_{1}\right) I_{k_{i}}\right)_{2 \leqslant i \leqslant N}$. Observe that

$$
\begin{aligned}
& \left(\begin{array}{cc}
I_{k_{1}} & 0 \\
-\Delta b T_{\{, i\}}^{-1} & \Delta
\end{array}\right) T_{\left\{y_{i}\right\}_{i \in X}}\left(\begin{array}{cc}
I_{k_{1}} & -T_{\left\{y_{i}\right\}}^{-1} \bar{b}^{-\mathrm{T}} \bar{\Delta} \\
0 & \bar{A}
\end{array}\right) \\
& =\left(\begin{array}{cc}
T_{\left\{\gamma_{i}\right\}} & 0 \\
0 & \Delta T_{\left\{\gamma_{i}\right\}_{2} \leqslant i \leqslant N} \bar{\Delta}-\Delta b T_{\left\{\gamma_{i}\right\}}^{-1} \bar{b}^{\mathrm{T}} \bar{\Delta}
\end{array}\right) .
\end{aligned}
$$

We prove now that the $(2,2)$ block of Eq. (26) coincides with $T_{\left\{y_{i}\right\}_{2 \leqslant i \leqslant 1}}$. In fact, the $(i, j)$ th block of $\Delta T_{\left\{y_{i}\right\}_{2 \leqslant s<i}} \bar{\Delta}-\Delta b T_{\left\{y_{i\}}\right.}^{-1} \bar{b}^{\mathrm{T}} \bar{\Delta}$ equals $\frac{\left(\bar{\lambda}_{i}+\lambda_{1}\right)\left(\lambda_{j}+\bar{\lambda}_{1}\right)}{\bar{\lambda}_{i}+\lambda_{j}} \bar{V}_{i}^{\mathrm{T}} J V_{j}-\bar{V}_{i}^{\mathrm{T}} J_{i} T_{\left\{y_{i}\right\}}^{-1} \bar{V}_{i}^{\mathrm{T}} J V_{j}$,

and, since the $(i, j)$ th block of $T_{\{i,\}_{2 \leqslant i \leqslant}}$ is given by Eq. (24), this proves the claim. Note also from Eq. (26) that $T_{\left\{x_{i}\right\}_{i \in X}}>0$ implies $T_{\left\{\gamma_{1}\right\}_{2 \leqslant \leqslant i}}>0$. Therefore, there exists a representation of the MPUM for $\left\{\mathscr{Y}_{i}^{\prime}\right\}_{2 \leqslant i \leqslant N}$ of the form

$$
\left(\begin{array}{cc}
-D^{\prime *} & N^{\prime *} \\
Q^{\prime} & -P^{\prime}
\end{array}\right)
$$

satisfying (2a)-(2f). The MPUM for $\left\{\mathscr{Y}_{i}\right\}_{i \in \underline{N}}$ is represented by

$$
\left(\begin{array}{cc}
-D^{*} & N^{*} \\
Q & -P
\end{array}\right):=\left(\begin{array}{cc}
-D^{\prime *} & N^{*} \\
Q^{\prime} & -P^{\prime}
\end{array}\right)\left(\begin{array}{cc}
-D_{1}^{*} & N_{1}^{*} \\
Q_{1} & -P_{1}
\end{array}\right) .
$$


We now show thar: Eq. (29) satisfies (2a)-(2f). In order to prove that $P$ is nonsingular, note from $\mathrm{Eq}$. (29) that $-P=Q^{\prime} N_{1}^{*}+P^{\prime} P_{1}$. Since $\left\|P^{\prime-1} Q^{\prime}\right\|_{\infty}<1$ and $\left\|N_{1}^{*} P_{1}^{-1}\right\|_{\infty}<1$ by inductive assumption, the matrix $P^{\prime-1} Q^{\prime} N_{1}^{*} P^{-1}+I=P^{\prime-1}(-P) P_{1}^{-1}$ is nonsingular on the imaginary axis, and the claim follows. The nonsingularity of $D$ is proved as follows. Observe from Eq. (29) that $-D^{*}=D^{\prime *} D_{1}^{*}+N^{\prime *} Q_{1}$. Note also from Eq. (15) that $Q_{1}=-N_{1}$, and therefore $-D=D_{1} D^{\prime}-N_{1}^{*} N^{\prime}=D_{1}\left(I-D_{1}^{-1} N_{1}^{*} N^{\prime} D^{\prime-1}\right) D^{\prime}$. Note that $\left\|N^{\prime} D^{\prime-1}\right\|_{\infty}<1$ by inductive assumption and also that $\|\left. D_{1}^{-1} N_{1}^{*}\right|_{\infty}<1$ because $R_{1}^{*} J R_{1}-P P^{*} J$, with $R_{1}$ the matrix in (15). There follows that $\left(I-D_{1}^{-1} N_{1}^{*} N^{\prime} D^{\prime-}\right.$ ) is nonsingular on the imaginary axis. This implies that $D$ is non-singular.

In order to prove (2b), note that $Q D-P N$ is the $(2,1)$ block of

$$
\left(\begin{array}{cc}
-D^{*} & N^{*} \\
Q & -P
\end{array}\right) J\left(\begin{array}{cc}
-D & Q^{*} \\
N & -P^{*}
\end{array}\right)
$$

Since Eq. (29) holds, and the representations of Eqs. (28) and (15) satisfy ( $2 \mathrm{~d})$ by inductive assumption, the claim follows easily.

We now prove that $P$ is Hurwitz. Assume by contradiction that there exist $\mu \in \mathbb{C}_{+}^{0}$ and a nonzero $v \in \mathbb{C}^{p}$ such that

$$
P(\mu) v=\left(\begin{array}{ll}
Q^{\prime}(\mu) & \left.-P^{\prime}(\mu)\right)
\end{array}\left(\begin{array}{c}
N_{1}^{*}(\mu) \\
-P_{1}(\mu)
\end{array}\right) v=0 .\right.
$$

Since $\left\|N_{1}^{*} P_{1}^{-1}\right\|_{H_{\infty}}<1$ and $P_{1}$ is Hurwitz, for every $\mu \in \mathbb{C}_{+}^{0}$ and every $v \in \mathbb{C}^{p}$ there holds $\left\|N_{1}^{*}(\mu) v\right\|_{2}^{2}<$ $\left\|P_{1}(\mu) v\right\|_{2}^{2}$; but then Eq. (31) contradicts the fact that $P^{\prime-1} Q^{\prime}$ is contractive. Therefore $P$ is Hurwitz.

Claim of (2d) can be proved by a straightforward computation, using Eq. (29) and the inductive assumption.

Let us prove $(2 a)$. It has been proved above that $P$ and $D$ are nonsingular and that $Q D-P N=0$; there follows $P^{-1} Q=N D^{-1}$. Therefore, we show that $D^{*} D-N^{*} N>0$ on the imaginary axis. Note that $D^{*} D-N^{*} N$ is the $(1,1)$ block of

$$
\begin{aligned}
& \left(\begin{array}{cc}
-D^{\prime *} & N^{\prime *} \\
Q^{\prime} & -P^{\prime}
\end{array}\right)\left(\begin{array}{cc}
-D_{1}^{*} & N_{1}^{*} \\
Q_{1} & -P_{1}
\end{array}\right) \\
& \quad \times J\left(\begin{array}{cc}
-D_{1} & Q_{1}^{*} \\
N_{1} & -P_{1}^{*}
\end{array}\right)\left(\begin{array}{cc}
-D^{\prime} & Q^{\prime *} \\
N^{\prime} & -P^{\prime *}
\end{array}\right)
\end{aligned}
$$

and by $(2 \mathrm{~d})$, this block equals $p(\mathrm{i} \omega) p^{*}(\mathrm{i} \omega) I_{m}>0$ for every $\omega \in \mathbb{R}$. The proof of (2f) follows a similar argument, since $N N^{*}-P^{*} P$ is the $(2,2)$ block of

$$
\begin{aligned}
& \left(\begin{array}{cc}
-D_{1} & Q_{1}^{*} \\
N_{1} & -P_{1}^{*}
\end{array}\right)\left(\begin{array}{cc}
-D^{\prime} & Q^{\prime *} \\
N^{\prime} & -P^{\prime *}
\end{array}\right) \\
& \quad \times J\left(\begin{array}{cc}
-D^{\prime *} & N^{\prime *} \\
Q^{\prime} & -P^{\prime}
\end{array}\right)\left(\begin{array}{cc}
-D_{1}^{*} & N_{1}^{*} \\
Q_{1} & -P_{1}
\end{array}\right) .
\end{aligned}
$$

This concludes the proof of $(1) \Rightarrow(2)$.

Let us now prove $(2) \Rightarrow(3)$. Let $(Q-P)=$ $F(U-Y)$, with $F$ a greatest common left divisor of $Q$ and $P$. We will show that $(U-Y)$ is a solution to the SNIP. Note that since $P$ is Hurwitz, $F$ is also Hurwitz. Since by assumption $P^{-1} Q=Y^{-1} U$ satisfies the metric constraint, we only have to show that $(U-Y)$ is such that $\left(U\left(\lambda_{i}\right)-Y\left(\lambda_{i}\right)\right) v=0 \forall v \in \mathscr{V}_{i}$, $i \in \underline{N}$. Since Eq. (13) is unfalsified on the data set $\mathscr{D}$, there follows

$$
\begin{aligned}
& \left(Q\left(\lambda_{i}\right)-P\left(\hat{\lambda}_{i}\right)\right) \\
& \quad=F\left(\lambda_{i}\right)\left(U\left(\lambda_{i}\right)-Y\left(\lambda_{i}\right)\right) v=0
\end{aligned}
$$

$\forall v \in \mathscr{V}_{i}, \forall i \in \underline{N}$. Since $F$ is Hurwitz, $F\left(\hat{\lambda}_{i}\right)$ is nonsingular for $i \in \underline{N}$, and the claim follows.

To prove $(3) \Rightarrow(1)$, denote with $(U-Y)$ a solution to the SNIP. Since $U$ and $Y$ are left coprime, there exist right coprime matrices $Z$ and $X$ with $X$ nonsingular such that $U X-Y Z=0$. This implies $Y^{-1} U=Z X^{-1}$ and since $\left\|Y^{-1} U\right\|_{H_{\infty}}<1$, for every trajectory $w \in \mathscr{L}_{2}\left(\mathbb{R}, \mathbb{C}^{q}\right)$ there holds $\|Z(\mathrm{~d} / \mathrm{d} t) w\|_{\mathscr{L}_{2}\left(\mathbb{R}, \mathbb{C}^{q}\right)}<\|X(\mathrm{~d} / \mathrm{d} t) w\|_{\mathscr{L}_{2}\left(\mathbb{R}, \mathbb{C}^{q}\right)}$. Therefore, the QDF induced by $\Phi(\zeta, \eta)=\bar{X}(\zeta)^{\mathrm{T}} X(\eta)$ $-\bar{Z}(\zeta)^{\mathrm{T}} Z(\eta)$ is averagely positive. We now show that this implies the positivity of $T_{\left\{y_{i}\right\}_{i \in \underline{\underline{X}}}}$. By resorting to the Smith form of

$\left(\begin{array}{l}X \\ Z\end{array}\right)$

it is easy to show that for every $i \in \underline{N}$ there exists a matrix $L_{i} \in \mathbb{C}^{m \times k_{i}}$ such that

$\left(\begin{array}{c}X(\mathrm{~d} / \mathrm{d} t) \\ Z(\mathrm{~d} / \mathrm{d} t)\end{array}\right) L_{i} \exp _{\lambda_{i i}}=V_{i} \exp _{\lambda_{i}}$.

Consider now a trajectory $a=\sum_{i=1}^{N} L_{i} x_{i} \exp _{i_{i}}$, where $\alpha_{i} \in \mathbb{C}^{k_{i} \times 1}$. It is easy to see that

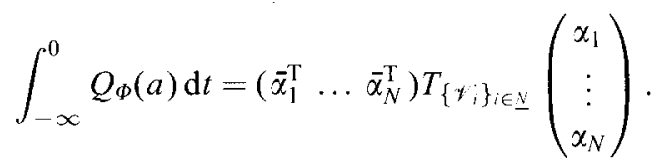

The trajectory $a$ is not of compact support, but an approximation argument yields that Eq. (36) is positive. Since the $\alpha_{i}$ 's are arbitrary, there follows $T_{\left\{\psi_{i}\right\}_{i \in \underline{N}}}>0$. 
Remark 4.1. The proof of implication (1) $\Rightarrow$ (2) in Theorem 4.1 suggests the following algorithm for computing a solution to the SNIP.

Input: $\lambda_{i} \in \mathbb{C}_{+}, y_{i}$ subspace of $\mathbb{C}^{q}, i \in \underline{N}$; assume that $\mathscr{V}_{i}$ is contractive, $V_{i}$ is a full column rank matrix such that $\operatorname{Im} V_{i}=\mathscr{Y}_{i}$, and $T_{\left\{\gamma_{i}\right\}_{i \in}}>0$.

Output: $(U, Y)$ solution to the SNIP

- $R_{0}=I_{q}$;

- $V_{k}^{\prime}=R_{k-1}\left(\lambda_{k}\right) V_{k}$

- $E_{k}=\left(\xi+\bar{\lambda}_{k}\right) I_{q}-V_{k}^{\prime} T_{\left\{{ }_{k}{ }_{k}\right\}}^{-1} \bar{V}_{k}^{\prime \top} J$

- $(Q-P)=\left(0_{p \times m} I_{p}\right) R_{N}$

- Compute left coprime $(U, Y)$ such that $Y^{-1} U=$ $P^{-1} Q$.

This procedure can be considered as the behavioral counterpart of the classical Nevanlinna recursive scheme for computing a solution to the scalar Nevanlinna interpolation problem (see $[6, p .165]$ ).

Remark 4.2. The structure of the representation given by Eq. (13) of the MPUM for 2 is close to that of the $J$-contractive rational matrix $\Theta$ at the core of the usual approach to metric interpolation problems (see [2, p. 386]). To illustrate this relationship, we will consider the tangential Nevanlinna interpolation problem $\left(k_{i}=1\right.$ for every $\left.i \in \underline{N}\right)$. In this case the $\Theta$ matrix is a $q \times q$ rational matrix such that

(a) $\Theta$ is analytic in $\mathbb{C}_{+}^{0}$;

(b) $\Theta^{*} J \Theta=J$ on the imaginary axis, and $\Theta^{*} J \Theta \leqslant J$ on $\mathbb{C}_{+}$;

(c) $\operatorname{Ker}\left(\Theta\left(\lambda_{i}\right)\right)=\mathscr{Y}_{i}$ for every $i \in \underline{N}$, where $\mathscr{V}_{i}$ is a one-dimensional subspace;

(d) $\lambda_{i}$ is a zero of multiplicity one for $\operatorname{det}(\Theta)$ for every $i \in N$.

We will show how to compute a rational matrix satisfying (a) - (d) above from a particular representation of the MPUM for $\mathscr{D}$. Observe that $\operatorname{since} \operatorname{dim}\left(\mathscr{V}_{i}\right)=1$, $i \in \underline{N}$, there follows $\operatorname{dim}\left(\mathscr{V}_{i}^{\perp}\right)=q-1$, and therefore the determinant of any kernel representation of the MPUM for $\mathscr{C}$ equals $\prod_{i=1}^{N}\left(\xi+\bar{\lambda}_{i}\right)^{q-1}\left(\xi-\lambda_{i}\right)$. We now show that there exists a representation $R$ of the MPUM for $\mathscr{Z}$ that satisfies (2a) (2f) of Theorem 4.1 and is row proper. In fact, for $N=1$, a representation for the MPUM is given by Eq. (14), with leading row coefficient matrix equal to the identity. Assume now that for $j \in N-1$ there exists a representation $R_{j}$ of the MPUM for the first $j$ subspaces and their duals, satisfying (2a)-(2f) of Theorem 4.1 and with leading row coefficient matrix equal to $I_{q}$. A representation of the MPUM for $N$ subspaces is obtained by premulti- plying $R_{N-1}$ by a representation $E_{N}$ of the MPUM for the $N$ th error subspace and its dual. This representation $E_{N}$ can be chosen to have leading row coefficient matrix equal to the identity. It is easy to see that the leading row coefficient matrix of $E_{N} R_{N-1}$ is also the identity. Note that this implies that there exists a representation $R$ of the MPUM for $\mathscr{T}$ satisfying (2a)-(2f) of Theorem 4.1 such that every entry is a polynomial of degree lower than or equal to $q$.

Consider now the rational matrix

$\Theta(\xi)=\frac{1}{\prod_{i=1}^{N}\left(\xi+\bar{i}_{i}\right)^{q}} R(\xi)$

we now show that it satisfies (a) - (d) above. Observe that each entry of Eq. (37) is a proper rational function with denominator $\prod_{i=1}^{N}\left(\xi+\bar{i}_{i}\right)^{q}$ and therefore is analytic in $\mathbb{C}_{+}^{0}$. Since the representation for the MPUM satisfies $(2 \mathrm{~d})$ of Theorem 4.1 , it is easily seen that $\Theta J \Theta=J$ for every point in $\mathbb{C}_{+}^{0}$. Observe also that $\operatorname{Ker}\left(\Theta\left(\hat{\lambda}_{i}\right)\right)=\operatorname{Ker}\left(R\left(\lambda_{i}\right)\right)=y_{i}, i \in \underline{N}$. Finally, note that $\operatorname{det}(\Theta)=\prod_{i=1}^{N}\left(\xi-\lambda_{i}\right) /\left(\zeta+\bar{\lambda}_{i}\right)$, whose only zeros are the $\lambda_{i}$ 's, each of multiplicity one.

\section{Characterization of all solutions to the SNIP}

In this section we provide a characterization of all solutions to the SNIP in terms of a representation given by Eq. (13) of the MPUM for 2 satisfying (2a)-(2f) of Theorem 4.1. The main result of this section is the following.

Theorem 5.1. Let a kernel representation of the MPUM for $\mathcal{I}$ be given as in Theorem 4.1. Then $(U-Y)$ is a solution to the SNIP if and only if there exist $\Pi \in \mathbb{C}^{p \times m}[\xi], \Phi \in \mathbb{C}^{p \times p}[\xi]$, and $F \in \mathbb{C}^{p \times p}[\xi]$ with $F, \Phi$ Hurwitz and $\left\|\Phi^{-1} \Pi\right\|_{H_{\infty}}<1$, such that

$F(U-Y)=(I-\Phi)\left(\begin{array}{cc}-D^{*} & N^{*} \\ Q & -P\end{array}\right)$.

Proof. Let us first prove sufficiency, assuming without loss of generality that $U$ and $Y$ are left coprime. Note that since $F(U-Y)$ is a left multiple of the MPUM for $\mathscr{L}$, it is unfalsified by $\mathscr{Z}$ and in particular

$$
\begin{gathered}
F\left(\lambda_{i}\right)\left(U\left(\lambda_{i}\right)-Y\left(\lambda_{i}\right)\right) v=0 \\
\forall v \in \mathscr{H}_{i} \text { and } \forall i \in \underline{N} .
\end{gathered}
$$

Since $F$ is Hurwitz, $F\left(\lambda_{i}\right)$ is nonsingular for all $i \in \underline{N}$; this, together with Eq. (39), implies that $(U-\bar{Y})$ is unfalsified by $\mathscr{V}_{i} \exp _{\lambda_{i}}, i \in \underline{N}$. We now prove 
that $\left\|Y^{-1} U\right\|_{H_{\infty}}<1$. In order to do this, we first show that $Y$ is Hurwitz. Note from Eq. (38) that $-F Y=\Pi N^{*}+\Phi P$. Assume by contradiction that $Y$ is not Hurwitz; then there exists a $\mu \in \mathbb{C}_{+}^{0}$ and a nonzero $v \in \mathbb{C}^{p}$ such that

$$
\begin{aligned}
-F(\mu) Y(\mu) v & =\left[\Pi(\mu) N^{*}(\mu)+\Phi(\mu) P(\mu)\right] v \\
& =0 .
\end{aligned}
$$

Since $P$ and $\Phi$ are Hurwitz, $\Phi(\mu)$ and $P(\mu)$ are nonsingular. Therefore from Eq. (40) we have

$$
\left[\Phi(\mu)^{-1} \Pi(\mu) N^{*}(\mu) P(\mu)^{-1}+I_{p}\right] v^{\prime}=0
$$

where $v^{\prime}=P(\mu) v$. The assumptions $\left\|N^{*} P^{-1}\right\|_{H_{\infty}}<1$ and $\left\|\Phi^{-1} \Pi\right\|_{H_{\infty}}<1$ imply

$\left\|P^{-1}(\mu) Q(\mu) \Phi^{-1}(\mu) \Pi(\mu)\right\|<1$

and consequently $\Phi(\mu)^{-1} \Pi(\mu) N^{*}(\mu) P(\mu)^{-1}+I_{p}$ is nonsingular. But this contradicts Eq. (41).

To prove that $\left\|Y^{-1} U\right\|_{\infty}<1$, we show that $U(\mathrm{i} \omega) U^{*}(\mathrm{i} \omega)-Y(\mathrm{i} \omega) Y^{*}(\mathrm{i} \omega)<0$ for all $\omega \in \mathbb{R}$. Observe that the representation for the MPUM for $\mathscr{D}$ satisfies (2d) of Theorem 4.1, and therefore

$$
\begin{aligned}
& F(\mathrm{i} \omega)(U(\mathrm{i} \omega)-Y(\mathrm{i} \omega)) J\left(\begin{array}{c}
U^{*}(\mathrm{i} \omega) \\
-Y^{*}(\mathrm{i} \omega)
\end{array}\right) F^{*}(\mathrm{i} \omega) \\
& =p(\mathrm{i} \omega) p^{*}(\mathrm{i} \omega)\left(\Pi(\mathrm{i} \omega) \Pi^{*}(\mathrm{i} \omega)-\Phi(\mathrm{i} \omega) \Phi^{*}(\mathrm{i} \omega)\right)
\end{aligned}
$$

for some Hurwitz polynomial $p$. Since $\left\|\Phi^{-1} \Pi\right\|_{\infty}$ $<1$, the right-hand side of Eq. (43) is negative definite for all $\omega \in \mathbb{R}$ and this, together with $Y$ Hurwitz, implies that $\left\|Y^{-1} U\right\|_{H_{\infty}}<1$.

Let us now prove necessity of the claim. Given a solution $(U-Y)$ of the SNIP, define $p(\xi)=$ $\prod_{i=1}^{N}\left(\xi+\bar{\lambda}_{i}\right)$ and $F:=p I_{p}$. Then $F(U-Y)$ is unfalsified by $\mathscr{D}$ and there:ore there exist $\Pi$ and $\Phi$ such that Eq. (38) holds. We now prove that $\left\|\Phi^{-1} \Pi\right\|_{\infty}<1$. Note that

$$
\begin{aligned}
& F(\mathrm{i} \omega)\left(U(\mathrm{i} \omega) U^{*}(\mathrm{i} \omega)-Y(\mathrm{i} \omega) Y^{*}(\mathrm{i} \omega)\right) F^{*}(\mathrm{i} \omega) \\
& \quad=p(\mathrm{i} \omega) p^{*}(\mathrm{i} \omega)\left(\Pi(\mathrm{i} \omega) \Pi^{*}(\mathrm{i} \omega)-\Phi(\mathrm{i} \omega) \Phi^{*}(\mathrm{i} \omega)\right) .
\end{aligned}
$$

Recall that $\left\|Y^{-1} U\right\|_{H_{\infty}}<1$ and that $F$ is Hurwitz; there follows that the left-hand side of Eq. (44) is negative definite on the imaginary axis, and therefore that $\Pi(\mathrm{i} \omega) \Pi^{*}(\mathrm{i} \omega)-\Phi(\mathrm{i} \omega) \Phi^{*}(\mathrm{i} \omega)<0$ for all $\omega \in \mathbb{R}$. We now prove that $\operatorname{det}(\Phi)$ has no roots on the imaginary axis. In fact, if $\operatorname{det} \Phi(\mathrm{i} \omega)=0$ for some $\omega \in \mathbb{R}$, there exists $v \in \mathbb{C}^{p}$ such that $\bar{v}^{\mathrm{T}} \Phi(\mathrm{i} \omega) v=0$ and therefore

$$
\begin{aligned}
& \bar{v}^{-\mathrm{T}}\left(\Pi(\mathrm{i} \omega) \Pi^{*}(\mathrm{i} \omega)-\Phi(\mathrm{i} \omega) \Phi^{*}(\mathrm{i} \omega)\right) v \\
& \quad=\|\Pi(\mathrm{i} \omega) v\|_{2}^{2}<0,
\end{aligned}
$$

which is a contradiction. This concludes the proof that $\left\|\Phi^{-1} \Pi\right\|_{\infty}<1$.

Consider now the biproper rational matrix $I_{p}+\alpha \Phi^{-1} \Pi N^{*} P^{-1}$, where $\alpha \in \mathbb{R}$ satisfies $0 \leqslant \alpha \leqslant 1$. Observe that contractiveness of $N^{*} P^{-1}$ and of $\Phi^{-1} \Pi$ implies that $I_{p}+\alpha \Phi^{-1} \Pi N^{*} P^{-1}$ is invertible on the imaginary axis for all $0 \leqslant \alpha \leqslant 1$; note that this also implies that $I_{p}+\chi \Phi^{-1} \Pi N^{*} P^{-1}$ is nonsingular as a rational matrix.

Consider now the rational matrix function $(-F Y)^{-1}$ $=(-p Y)^{-1}$. From Eq. (38) follows $-p Y=\Phi P+$ $\Pi N^{*}$, and therefore

$$
\begin{aligned}
(-p Y)^{-1} & =\left[\Phi\left(I+\Phi^{-1} \Pi N^{*} P^{-1}\right) P\right]^{-1} \\
& =P^{-1}\left(I+\Phi^{-1} \Pi N^{*} P^{-1}\right)^{-1} \Phi^{-1} .
\end{aligned}
$$

Evidently there holds

$$
\begin{aligned}
& \operatorname{det}\left((-p Y)^{-1}\right) \\
& \quad=\operatorname{det}\left(P^{-1}\right) \operatorname{det}\left(\left(I+\Phi^{-1} \Pi N^{*} P^{-1}\right)^{-1}\right) \operatorname{det}\left(\Phi^{-1}\right) .
\end{aligned}
$$

In order to prove that $\Phi$ is Hurwitz, we will prove that $1 / \operatorname{det} \Phi$ has no poles in $\mathbb{C}_{+}$by computing its winding number wno( $(1 / \operatorname{det} \Phi)$ from the expression given by Eq. (47). Observe that from the fact that $U$ and $Y$ are coprime and $\left\|Y^{-1} U\right\|_{H_{\infty}}<1$ there follows $-p Y$ is a Hurwitz polynomial matrix. Therefore, wno $\left(\operatorname{det}\left((-p Y)^{-1}\right)\right)$ is zero. Applying the logarithmic property of the winding number function and the fact that $P$ is a Hurwitz polynomial matrix, there follows from (47) that

$$
\begin{aligned}
0= & w n o\left(\operatorname{det}\left(P^{-1}\right)\right)+w \operatorname{wno}\left(\operatorname{det}\left(I+\Phi^{-1} \Pi N^{*} P^{-1}\right)^{-1}\right) \\
& +w n o\left(\operatorname{det}\left(\Phi^{-1}\right)\right) \\
= & w n o\left(\operatorname{det}\left(I+\Phi^{-1} \Pi N^{*} P^{-1}\right)\right) \\
& +w n o\left(\operatorname{det}\left(\Phi^{-1}\right)\right) .
\end{aligned}
$$

Note that wno $\left(\operatorname{det}\left(I+\alpha \Phi^{-1} \Pi N^{*} P^{-1}\right)\right)$ is a continuous function of $\alpha$ taking integer values, and therefore that its value is independent of $\alpha$; this implies that $w n o\left(\operatorname{det}\left(I+\alpha \Phi^{-1} \Pi N^{*} P^{-1}\right)\right)=w n o(\operatorname{det}(I))=$ 0 for all $0 \leqslant x \leqslant 1$. Then from Eq. (48) we have wno $\left(\operatorname{det}\left(\Phi^{-1}\right)\right)=0$, and this implies that the number of zeros of $\operatorname{det}(\Phi)$ in $\mathbb{C}_{+}$is zero. This concludes the proof. 
Remark 5.2. The characterization Eq. (38) of all solutions to the SNIP bears close resemblance to the characterization of the solutions to the tangential Nevanlinna interpolation problem given in the linear fractional transformation setting (see [2, p. 386]).

\section{Conclusions}

The main results of this paper are Theorems 4.1 and 5.1 that bring together the MPUM and the calculus of QDFs to provide a behavioral point of view on the solvability of the SNIP and on the characterization of all its solutions. This interpretation allows to compute a solution to the SNIP by recursively computing the MPUM of a set of data with a special structure.

\section{References}

[1] A.C. Antoulas, J.C. Willems, A behavioral approach to linear exact modelling, IEEE Trans. Automat. Control 38 (1993) 1776-1802.

[2] J.A. Ball, I. Gohberg, L. Rodman, Interpolation of Rational Matrix Functions, Birkhäuser, Berlin, 1990.

[3] J.C. Doyle, B.A. Francis, A.R. Tannenbaum, Feedback Control Theory, McMillan, New York, 1992.

[4] I.P. Fedčina, A criterion for the solvability of the NevanlinnaPick tangent problem, Mat. Issled 7 (1972) 213-227.
[5] P.A. Fuhrmann, A polynomial approach to Hankel norm and balanced approximations, Linear Algebra Appl. 146 (1991) 133-220.

[6] J.B. Garnett, Bounded Analytic Functions, Academic Press, New York, 1963.

[7] J.W. Helton, The distance of a function to $H^{x}$ in the Poincare metric; electrical power transfer, J. Funct. Anal. 38 (1980) 273-314.

[8] H. Kimura, Robust stabilizability for a class of transfer functions, IEEE Trans. Automat. Control 29 (1984) $788-793$.

[9] R. Nevanlinna, Über beschränkte Funktionen, die in gegeben Punkten vorgeschrieben Werte annehmen, Ann. Acad. Sci. Fenn. Ser. A I Mat. Dissertationes 13 (1919).

[10] G. Pick, Über die Beschränkungen analytische Funktionen. welche durch vorgegebene Funktionswerte bewirkt werden, Math. Ann. 77 (1916) 7-23.

[11] J.C. Willems, From time series to linear system - Part I. Finite dimensional linear time-invariant systems, Automatica 22 (1986) $561-580$.

[12] J.C. Willems, From time series to linear system -- Part II. Exact modelling. Automatica 22 (1986) 675-694.

[13] J.C. Willems, Paradigms and puzzles in the theory of dynamical systems, IEEE Trans. Automat. Control 36 (1991) 259-294.

[14] J.C. Willems, H.L. Trentelman, On quadratic differential forms, SIAM J. Control Optim., submitted.

[15] J.C. Willems, H.L. Trentelman, $H_{\infty}$ control in a behavioral context, Part I: The full information case, IEEE Trans. Automat. Control, submitted.

[16] D.C. Youla, M. Saito, Interpolation with positive-real functions, J. Franklin Inst. 284 (1967) 77-108. 